\title{
Etiología Bacteriana de la Diarrea Aguda del Lactante en Otoño e Invierno
}

\author{
Dr. Mario Henríquez 0.1; Dr. Guillermo Venegas V. ${ }^{2}$; Dr. Gonzalo Soto T. ${ }^{3}$; \\ Dra. Tatiana Rudolff F.4; Dr. Julio Salas C. 3 .
}

\author{
Bacteriae in infants with acute diartheic syndrome during cold \\ months of the year
}

\begin{abstract}
The bacteriological findings from a prospective study on children aged under 2 years admitted to hospital with acute diarrhoea in the cold seasons of the year are described. The study periods extend from March 21 to September 21 in the years 1983 and 1984. Samples of stool were obtained at admission to the hospital, Bacteriological investigations were done at the Department of Microbiology in the University of Concepcion. During 1983, 201 patients were investigated. Putative pathogenic bacteriae were identilicd in 30 children (15\%). The comparative frecuency of isolation of Enteropathogenic E. coli, Shigella. Salmonella, and Campylobacter jejuni were $9.5 \% 3.5 \%$ $1.5 \%$ and $0.5 \%$ respectively. Yersinia enterocolitica was not found in the first year of the study. During 1984,140 - patients were investigated. Enteropathogentc bacteriae were isolated from the stools of 28 children (20\%). The comparative frecuency of isolation of Enteropathogenic E. coli, Shigella, Salmonella, Campylobacter jejuni. and Yersinia enterocolitica were $10 \%, 4.3 \% 1.4 \% 2.9 \%$ and $1.4 \%$ respectively. Children in which bacteriae were isolated also had undernutrition in $50 \%$ and $43 \%$ of the cases in the years 1983 and 1984 respectively.

(Key words: Acute diarrhoea. Bacterial diarrhosa. Cold seasons).
\end{abstract}

La diarrea aguda del lactante sigue siendo en nuestro país un problema importante de salud ${ }^{1}$. Diversos estudios epidemiológicos nacionales demuestran una etiología infecciosa en más de la mitad de los casos, manteniendo los enteropatógenos bacterianos, aún en ćpocas invernales, una significativa frecuencia de aislamiento $0^{2-4}$. En esta situación convicne efectuar estudios periódicos de prevalencia de distintos agentes en diferentes áreas, para configurar el espectro local y tomar las respectivas medidas epidenuológicas y terapéuticas.

El propósito de este estudio fue medir la frecuencia de aislamiento bacterianoen ила muestra representativa de lactantes hospitalizados por diarrea aguda y establecer la prevalencia de los diferentes agentes enteropatógenos en nuestro

1. Departamento de Microbiologia. Facultad de Ciencias Biológicas y Recursos Naturales, Universidad de Concepción.

2. Departamento de Pediatría. Universidad de Concepción.

3. Residente becario. Depto. de Pediatría, Facultad de Medicina, Universidad de Concepción.

4. Servicio de Pediatría. Hospital Guillermo Grant Be. navente. Concepción.

Trabajo financiado pot la Dirección de Investigación de la Universidad de Concepción, Proyecto 20.35.03. medio. La investigación se desarrolló en forma prospectiva durante las estaciones frías de dos años consecutivos, 1983 y 1984.

\section{MATERIAL Y METODO}

Entre el 21 de marzo y el 21 de septiembre de 1983 y durante el mismo periodo de 1984 , se investigaron los lactantes ingresudos con el diagnóstico de diarrea aguda al Scrvicio de Pediatría del Hospital Guillermo Grant Benavente de Concepción. Los límites de edad fueron establecidos entre 1 y 24 meses, todos los pacientes sufrían deshidratación moderada o severá, ninguno tenia antecedentes recicntes de hospitalización. Se registró su procedencia y estado nutricional.

Al ingresar, se tomó de cuda lactantc, mediante sonda rectal estéril, una muestra de deposición, que fue transportada en medio de Cary Blair, para su procesamicnto, al Laboratorio de Microbiologia de la Universidad de Concepción. La sicmbra se efectuó en agar XLD y caldo tetrationato para cultivo de Shigella, Salmonella y Yersinia enterocolítica: en agar sangre y agar sorbitol para la investigación de Escherichia coli enteropatógeno y, en me dio Butzler, para cultivo de Campylobacter jejuni. La investigación bio- 
quimica y serológica de Salmonella y Shigella se efectuó mediante las técnicas recomendadas por Edwards y Ewing 5 . Las cepas de Yersinia enterocolítica obtenidas fueron confirmadas y serotipificadas en el Instituto Pasteur de París. La identificación de los serogrupos clásicos de $\mathrm{E}$. coli enteropatógeno se desarrolló mediante antisueros comerciales $O B$. Las colonias caracteristicas de Campylobacter fueron examinadas por tinción y sometidas a las pruebas de oxidasa y caralasa y además a la prueba de confirmación de tolerancia a la temperatura y crecimiento en clorhidrato de trifeniltetrazolio.

La sensibilidad a los antibióticos y quimioterapéuticos de las cepas enteropatógenas identificadas, fue determinada mediante pruebas de difu. sión en agar.

\section{RESULTADOS}

Entre el 21 de marzo y 21 de septiembre de 1983 se estudiaron 201 lactantes, aislándose enteropatógenos bacterianos en $15 \%$ de los casos. En el mismo período de 1984 se estudiaron 140 lactantes, en $20 \%$ de los cuales se aislaron bacterias potencialmente involucradas en la diarrea que sufrian.

La distríbuciôn geográfica de los lactantes con aislamientos bacterianos positivos estuvo influida por las normas administrativas y, en ambos periodos, no tuvo relación con brotes epidémicos. El $90 \%$ de los casos de 1983 tenía menos de 12 meses de edad, otro tanto ocurrió con $68 \%$ de los pacientes durante 1984. El 50\% de estos lactantes tenía algún grado de desnutrición en 1983, y $43 \%$ en 1984 .

La distribución de los enteropatógenos bacterianos aislados, según su frecuencia de aparición, se expone on la Tabla 1 .

Tabla 1

Disıribución de los enteropatógenos bacterianos aislados según su frecuencia, 1983 y 1984

\begin{tabular}{lcc}
\hline & $\begin{array}{c}1983 \\
(\mathrm{n}=201)\end{array}$ & $\begin{array}{c}1984 \\
(\mathrm{n}=140)\end{array}$ \\
\hline E. coli enteropatógeno & $19(9,5 \%)$ & $14(10,0 \%)$ \\
Shigella & $7(3,5 \%)$ & $6(4,3 \%)$ \\
Salmonella & $3(1,5 \%)$ & $2(1,4 \%)$ \\
Campylobacter jejuni & $1(0,5 \%)$ & $4(2,9 \%)$ \\
Yersinia enterocolítica & - & $2(1,4 \%)$ \\
\hline
\end{tabular}

En ambos períodos predomina E. coli enteropatógeno $(9,5 \%$ en 1983 y $10 \%$ en 1984), con menor prevalencia de.Shigella y de Salmonella.
En nuestro medio también aparecen Campylobacter jejuni $(0,5 \%$ en 1983 y $2,9 \%$ en 1984), y Yersinia enterocolítica $(1,4 \%$ en 1984) como agentes enteropatógenos.

La frecuencia de los serogrupos clásicos de $\mathbf{E}$. coli enteropatógenos se describe en la Tabla 2. Los serogrupos $0_{126}: B_{16}, 0_{111}: B_{4} y^{0_{119}}: B_{14}$ predominaron en 1983 . El serogrupo $0_{111}: B_{4}$ fue el más frecuente durante 1984, no observándose el serogrupo $0_{126}: B_{16}$ en este periodo.

Tabla 2

Distribución de los serogrupos de Escherichía coli enteropa tógeno de acuerdo a su frecuencia, 1983 y 1984

\begin{tabular}{lcc}
\hline Serogrupo & $\begin{array}{r}1983 \\
n=19\end{array}$ & $\begin{array}{r}1984 \\
n=14\end{array}$ \\
\hline $0_{26}: B_{6}$ & 1 & - \\
$0_{55}: B_{5}$ & 3 & 2 \\
$0_{86}: B_{7}$ & 1 & 1 \\
$0_{11}: B_{4}$ & 4 & 8 \\
$0_{119}: B_{14}$ & 4 & 1 \\
$0_{126}: B_{16}$ & 5 & 1 \\
$0_{127}: B_{8}$ & - & 1 \\
$0_{128}: B_{12}$ & 1 & 1 \\
\hline
\end{tabular}

En lo que respecta a las especies de Salmonella identificadas, hubo un caso de S. Typhy en 1983 y 2 en cada año de S. typhimurium.

Las especies de Shigella aparecen distribuidas en forma uniforme durante 1983 (S. Flexneri 2, S. boidii 2 y S. sonnei 3). En 1984 de los 6 casos con aislamiento de esta especic 5 eran $\mathrm{S}$. flexneri y IS. boydii.

Los dos casos de Yersinia enterocolítica identificados correspondieron a la misma cepa, qujmiotipo 4, scrotipo 0:3, lysotipo VIII.

El patrón de sensibilidad a los antimicrobianos de uso habitual, en las cepas de E. coli enteropatógeno, Shigella y Salmonella, se detalla en la Tabla 3. Todas las cepas de E. coli enteropatógenas eran sensibles a Amikacina; a Furazolidona en $97 \%$ a Cefotaxima en $97 \%$ y a Gentamicina en $82 \%$. La sensibilidad a Ampicilina existía sólo en $18 \%$ de las cepas estudiadas.

Las Shigella sp. resultaton sensibles a Gentamicina, Amikacina, Cefradina, Furazolidona, Sulfametoxazol-trime toprim y Cloramfenicol, observándose menor sensibilidad a Ampicilina.

Las 4 cepas de Salmonella typhimurium fueron sersibles a Furazolidona, Amikacina, Cefra. dina y Cefotaxima, mostrando menor sensibilidad a Ampicilina, Cloramfenicol y Gentamicina. Ninguna de ellas resultó sensible a Sulfame toxazol-Trime toprim.

Durante los dos períodos estudiados, $18 \%$ de los lactantes en que se identificó un enteropató- 
geno bacteriano mejoraron sin que hubiesen usado antibióticos en su hospitalización.

\section{Tabla 3.}

Sensibilidad in vitro de las cepas de E. coli enteropatógeno (ECE) Shigella species (SS) y Salmonella typhimutium (ST) a drogas antimicrobianas de empleo habitual.

$$
\operatorname{ECE}(n=33) \operatorname{SS}(n=13)
$$

Amikacina

Ampicilina

Cefotaxima

Cef radina

Cloramfenicol

Furazolidona"

Gentamicina

SMT . TMP

$\begin{array}{rr}33 & 13 \\ 6 & 7 \\ 32 & 13 \\ \mathrm{NP} & 13 \\ 21 & 10 \\ 32 & 12 \\ 27 & 13 \\ 20 & 12\end{array}$

SMT - TMP $=$ Sulfanctoxazol-Trime toprim $\mathrm{NP}=$ No probado.

\section{COMENTARIO}

La frecuencia con que se aislaron bacterias enteropatógenas en la diarrea aguda del lactante durante las estaciones frías del año, tue en nuestro estudio de dos años consecutivos, inferior a la observada por otros autores nacionales en épocas semejantes ${ }^{3-4}$.

En nuestra muestra, la mayor proporción de lactantes correspondió a menores de 1 año. Los lactantes portadores de enteropatógenos, presentaron cifras de desnutricion que excedicron signficativamente la estadistica nacional para esa edad.

E. coli enteropatógenas fueron los agentes bacterianos más frecuentemente aislados, resultando concordante con referencias latinoamericanas y nacionales ${ }^{3-4-6-7}$, que reitera nuestra diferencia con respecto a países industrializa$\operatorname{dos}^{\mathbf{B}-9}$. Asimismo, en el analisis de los scrogrupos, se mantiene la frecuencia observada en nuestro país en lactantes hospitalizados por diarrea, con predominio de $0_{111}: B_{4}, O_{119}: B_{14}$, $0_{55}: B_{5}$ y $0_{126}: B_{6}{ }^{3-4}$.

La incidencia de Shigellas en estaciones frías en esta muestra, fue similar a la comunicada por Prado y col. en un período semejante 4 . A diferencia de lo ocurrido en otros estudios nacionales durante períodos frios ${ }^{3-4}$, en esta serie se aislaron Salmonellas, aunque en una proporción menor a la encontrada en verano en los estudios citados.

El patrón de sensibilidad a los antimicrobianos, de las cepas aisladas de E. coli enteropatógeno, Shigella y Salmonella, fue semejante al de otros informes nacionales ${ }^{10}$, excepto por la aún menor sensibilidad de Salmonellas a Gentamicina, Ampicilina y Cloramfenicol y su falta de sensibilidad a Sulfametatoxazol-Trime troprim.

Yersinia enterocolítica ha emergido como un enteropatógeno importante en la infancia ${ }^{8-10-}$ 11-12. donde produce infecciones más frecuentemente en los meses de verano. En lactantes chilenos se ha comunicado una incidencia de I\% en los meses cálidos ${ }^{13}$. En nuestro estudio, durante dos periodos fríos se detectaron dos casos, ambos en 1984, con una incidencia de $1,4 \%$ en lá diarrea aguda del lactante durante ese período, en esta región. Ambos casos pertenecian al quimiotipo 4 , serotipo $0: 3$, reconocido como el más inmu nogénico y el más claramente asociado a infección 12 .

Campylobacter jejuni es un importante agente de diarrea bacteriana en la niñez, siendo tan frecuente como Salmonellas y Shigellas ${ }^{4-8-9-1-}$ 14-16. Su mayor incidencia se observa dwrante los meses calidos ${ }^{9-17}$. Existen estudios que sugieren que la prevalencia es mayor en los paises en desarrollo que en los países industrializa$\operatorname{dos}^{17}$. En Chile, se ha comunicado, en lactantes con diarrea, incidencias de $7,8 \%$ en verano y $9,5 \%$ en invierno ${ }^{4}$. En Concepción nuestro registro corresponde. en épocas frías, a cifras de $0,5 \%$ en 1983 y $2,9 \%$ en 1984 , superando en el último periodo a la de Salmonella.

En suma, durante las estaciones frías del año, los enteropatógenos bacterianos continúan teniendo importancia como causa de diarrea aguda del lactante. Asimismo, se puederi comprobar cambios de prevalencia que no deben ser ignorados.

\section{RESUMEN}

Se investigaron prospectivamente las causas bacterianas del sindrome de diarrea aguda en lactantes de 1 a 24 meses de edad, hospitalizados por esta causa en un hospital de Concepción. El estudio se hizo entre el 21 de Marzo y el 21 de Septiambre de 1983 y en el mismo período de 1984. Al ingresar se tomaron de zada paciente, muestras para cultivo de deposiciones. Se investigaron 201 pacientes en 1983, aislándose bacterias enteropatógenas de $30(15 \%)$. Se aisló E. coli enteropatógeno $(9,5 \%)$, Shigella $(3,5 \%)$, Salmonella $(1,5 \%)$ y Campylobacter jejuni $(0,5 \%)$. No se encontró Yersinia enterocolf́tica en ese año. En 1984 fueron investigados 140 pacientes, obteniendo resultados positivos en $28(20 \%)$. La frecuencia con que se aisló $E$. coli enteropatógeno fue $10 \%$, Shigelia $4,3 \%$, Salmonella $1,4 \%$, 
Campylobacter jejuni 2,9\% y Yersinia enterocolí. tica 1,4\%. Entre los lactantes en que se aislaron bacterias enteropatógenas, existía evidencia de algún grado de desnutrición en $50 \%$ y $43 \%$, respectivamente, para los periodos de 1983 y 1984.

\section{REFERENCIAS}

1. Anónimo: Seminario Control de Enfermedades Diareicas. Epidemiología y vigilancia de las enfermedades diarreicas 1980. Ministerio de Salud de Chile. Dpto. de Programa de las Personas.

2. Prodo V., Mimica I y Donoso E.: Estudio etiológico de la diarrea aguda del lactante. Aportes de la inmunofluorescencia. Estudio de sensibilidad in vitro. Rey. Chil. Pediatr. 45: 135, 1974.

3. Zacaias $J$., Spencer E., Prado V., Herskovic P. y col.: Recuperación fecal de rotavirus $y$ otros enteropatógenos en lactantes menores de 1 año con diarrea aguda. Rey. Chil. Pediatr. 53: 111, 1982.

4. Prado V., Braun S., Bosch $P$., Bercovich $M$., Reyes M. y Sawada $M$.: Análisis de Escherichia coli enteropatógeno chásico como causa endémica de diarrea aguda en niños chilenos. Rev. Chil. Pediatr. 55: 171, 1984.

5. Edwards P.R. and Ewing W.H.: Identification of Enterobacteriaceae. 3rd. Ed. Co. Minneapolis. Burgess Publishing Company, 1972.

6. Edelman $R$, and Levine $M$.: Summary of a workshop on enteropathogenic Escherichia coli. J. Infect. Dis. 147: 1108, 1983.
7. Toledo M.R., do Carno A., Murahovschi J., Ramos $S$. and Trabulsi L.: Enteropathogenjc Escherichia coli serotypes and endemic diarrhoea in infants. In. fect. lnmun. 39: 586, 1983.

8. Pai C.H., Sorger S., Lockman L., Sinai R. and Marks M.I.: Campylobacter gastroenteritis in children. $J$. Pediatr 94: 589, 1979.

9. San Joaquin V.H. and Welch D.F.: Campylobacter enteritis. Clin. Pediatr. 23: 311, 1984.

10. Prado V., Reyes L., UPbina M.R. y Phillips M.O.: Panorama actual de la resistencia de bacterias enteropatógenas a los antimicrobianos de uso habitual. Cursos Avances en Entermedades Infecciosas en Pediatría. Universidad Católica de Chile, Santiago, 1984.

11. Feigin R.D. and Cherry J.D.: Textbook of Pediatric Infectious Diseases. W.B. Saunders Company, Vol. 1 ; 833 and 1012,1981 .

12. Marks M.I. Pai C.H., Lafleur L., Lackman L. and Hommerberg $O$ : Yersinia enterocolitica gastroenteritis: A prospective study of clinical, bacteriologic, and epidemiologic features. J. Pediatr. 96:36, 1980 .

13. Prado V. y Cohen J.: Yersinia enterocotitica, su tol como agente de diarrea aguda en lactantes. Rev. Chil. Pediatr. 45: 69, 1980 .

14. Duffau G., Lagos R., Garcia J. y Maldonado A.* Campylobacter fetus eubespecie jejuni er lactantes con síndrome diartelico agudo. Rev. Chil. Pediatr. 53: 205,1982 .

15. Kormoh M.A. and Fleming P.C.: Campylobacter enteritis in children. J. Pediats, $94: 527,1979$.

16. Retig P.J.: Campylobacter infections in human beings. J. Pediatr. 94: 855, 1979

17. Bloser M.J. and Reller L.B.: Campylobacter enteritis. N. Eng. J. Med. 305: 1444, 1981. 